\title{
Zentralvorstandssitzung vom 18. April 2013
}

\begin{abstract}
Swiss eHealth Barometer - Die Befragung «Swiss eHealth Barometer» wurde dieses Jahr zum vierten Mal durchgeführt. Neben den Ärzten, den IT-Verantwortlichen der Spitäler und den Kantonen wurden neu auch die Apotheker sowie 1011 Stimmberechtigte befragt. Der Rücklauf bei den Ärzten war mit 20\% geringer als in der Vergangenheit (2012: 24\%). Bei den Ärzten manifestiert sich jedoch eine interessante Dynamik: Ein Drittel der Praxis- und Belegärzte gibt an, die Krankengeschichte vollständig elektronisch zu führen. Von diesen äussert wiederum ein Drittel, diese seit höchstens zwei Jahren zu nutzen. Auch wenn die Zahlen einem Bias unterliegen sollten, so kann dies gleichwohl als ein Indiz für die Einleitung eines Kulturwandels hin zur elektronischen Krankengeschichte interpretiert werden. Haupthindernisse für die Umstellung sind zusätzlicher Erfassungsaufwand und Abhängigkeiten von Softwarefirmen. Bei den Bürgern ist eher die Einstellung zur IT als die persönliche Erfahrung im Behandlungsumfeld massgebend für die Haltung gegenüber eHealth: Je vertrauter der eigene Umgang mit Informatikmitteln, desto geringer sind die Vorbehalte gegenüber eHealth, weil bei Gesundheitsfragen bereits heute das Internet alltäglich ist.
\end{abstract}

NAKO-Kodex - NewIndex hat zum Ziel, einen gesamtschweizerischen Datenpool bereitzustellen, unter anderem um Ärzten ein Instrument im Fall von Wirtschaftlichkeitsverfahren zur Verfügung zu stellen. NewIndex stellt die Datenaggregate für den Praxisspiegel zur Verfügung, der es dem einzelnen Arzt erlaubt, sich in einem Benchmark mit Kollegen der gleichen Fachrichtung zu vergleichen. Die anonymisierten, konsolidierten und aggregierten Daten, welche NewIndex in der sogenannten NAKO (Nationale Konsolidationsstatistik) auswertet und analysiert, sind seit 2004 unverzichtbar für die Tarifverhandlungen der FMH und der Kantonalen Ärztegesellschaften. Nun hat NewIndex den NAKO-Kodex überarbeitet, welcher die Datenverwendung regelt. Neu enthält der überarbeitete Kodex strengere Regelungen, und bei Missbrauch besteht die Möglichkeit einer Konventionalstrafe.

Kollektivmitgliedschaft von Arztpraxen - Gemäss AHV-Recht können Arbeitnehmer in Verbandsausgleichskassen wie z.B. der Medisuisse versichert werden, wenn der Arbeitgeber Mitglied in der Gründerorganisation der Verbandsausgleichskasse ist. Die Bedingung ist erfüllt, wenn der Arzt (oder die Ärzte einer Gruppenpraxis) als selbständig erwerbendes FMH-Mitglied Gründerorganisation von Medisuisse ist. Weil die Arztpraxis als Unternehmen bisher nicht Mitglied bei der FMH sein kann, ist die Bedingung nicht erfüllbar, wenn der Arbeitgeber eine Arztpraxis in Form einer juristischen Person (z. B. AG oder $\mathrm{GmbH}$ ) ist. Es ist anzustreben, dass sich FMHMitglieder unabhängig von der Frage der Rechtsform ihrer Arztpraxis bei Medisuisse versichern können. In den FMH-Statuten soll deshalb die Möglichkeit geschaffen werden, eine Kollektivmitgliedschaft von Arztpraxen in Ergänzung (nicht als Ersatz der Einzelmitgliedschaft der dort tätigen Ärzte) bei der FMH einzuführen.

\section{Findet Sie.} Das Ärzteverzeichnis doctorfmh.

\section{Dort präsent sein, wo Patienten ihren Arzt suchen. Kostenlos für Mitglieder.}

